\title{
FOREWORD TO THE SECOND EDITION
}

Ten years ago, Peter Del Tredici produced a very useful and thoughtful field guide to the common urban plants of the northeastern United States. The region includes some of our largest urban agglomerations and suburban territories, extending up and down the East Coast from Montreal to Washington, D.C., and from Boston to Detroit. That first edition provided an important resource for urban residents and land managers by giving them a convenient tool to identify and learn about the plants that inhabit their cities. It is a welcome event to mark the publication of a new edition of that pioneering book that adds forty-five new species to the plant roster-an increase of $20 \%$ over its predecessor.

Urban ecologists and climate scientists have become more convinced that the continued human movement of organisms to cities, along with changes in climate and associated local environmental conditions, have transformed them into "novel ecosystems." These new combinations of species have no analogue in the past and their future trajectories are unknown. On the one hand, this realization suggests that the plants that spontaneously establish in cities are capable of adapting to ongoing environmental change; on the other hand, it shows that managing the ecology of urban ecosystems strictly by looking backward is a poor strategy for keeping pace with our rapidly changing environment.

These conclusions may seem radical and, to some perhaps, disturbing. The new introduction to Wild Urban Plants of the Northeast, however, provides a concise and easily comprehensible summary of the key lessons of modern urban ecology that support these conclusions. It includes well-documented and thoughtful descriptions of the origins, characteristics, evolution, and benefits of plants that establish spontaneously in urban habitats. This introduction is, in fact, a very good survey in approachable language of relevant findings from modern urban ecology.

Readers of this book will garner much more understanding than simply knowing what plants are growing in their neighborhood or in the neglected slivers of land in their cities or towns. Readers will begin to appreciate more fully the role of ecology-the patterns and processes influenced by organisms of all sorts-in the city. They will also come to appreciate how much disturbance and intentional change takes place in cities, and how plants respond to these pressures. And finally, 
they will come to understand the immense amount of "free work" that spontaneous plants perform in urban systems. With this understanding, they will question the simplistic and judgmental focus on species labeled as exotic or invasive, and instead, will evaluate the resulting novel ecosystems by the functions and services they provide.

This book provides readers with a viable alternative to the usual simplistic labels of species as either native or non-native. Instead, they will find a rich section on the "Cultural Significance" of each species that illuminates the compelling connections between humans and the plants that surround them in the urban environmentmany of them dating back thousands of years. These human-plant interactions are continuing to evolve along with our changing climate, and readers will see how exciting an arena the cities are for understanding the ongoing process of plant evolution in an urbanized world. Specialists in ecological research who delve deeply into the book will find some very nice ideas to stimulate new research.

This new edition is important to a broad audience who already have an interest in plants, but it is also an important statement of the emerging and changing ecological reality of our cities. Wild Urban Plants of the Northeast is not just an updated tool for the task of identifying individual species of plants in cities and towns and appreciating their history. It is also an important tool to help land managers, policymakers, landscape architects, garden designers, maintenance contractors, and residents better understand, and thus better manage, novel urban ecosystems. Few tasks are more important for people who inhabit our increasingly urbanized world.

\author{
Steward T. A. Pickett \\ Cary Institute of Ecosystem Studies and \\ Baltimore Ecosystem Study \\ Millbrook, New York
}

